\title{
Bose-Einstein condensation in the pseudogap phase of cuprate superconductors
}

\author{
A. S. Alexandrov \\ Department of Physics, \\ Loughborough University, \\ Loughborough LE11 3TU, United Kingdom
}

\begin{abstract}
We have identified the unscreened Fröhlich electron-phonon interaction (EPI) as the most essential for pairing in cuprate superconductors as now confirmed by isotope substitution, recent angle-resolved photoemission (ARPES), and some other experiments. Low-energy physics is that of mobile lattice polarons and bipolarons in the strong EPI regime. Many experimental observations have been predicted or explained in the framework of our "Coulomb-Fröhlich" model, which fully takes into account the long-range Coulomb repulsion and the Fröhlich EPI. They include pseudo-gaps, unusual isotope effects and upper critical fields, the normal state Nernst effect, diamagnetism, the Hall-Lorenz numbers, and a giant proximity effect (GPE). These experiments along with the parameter-free estimates of the Fermi energy and the critical temperature support a genuine Bose-Einstein condensation of real-space lattice bipolarons in the pseudogap phase of cuprates. On the contrary the phase fluctuation (or vortex) scenario is incompatible with the insulating-like in-plane resistivity and the magnetic-field dependence of orbital magnetization in the resistive state of underdoped cuprates.
\end{abstract}

PACS numbers: 71.38.-k, 74.40.+k, 72.15.Jf, 74.72.-h, 74.25.Fy 


\section{INTRODUCTION}

Relatively high superfluid $T_{c}$ of ${ }^{4} \mathrm{He}(\approx 2.17 \mathrm{~K})$ compared with $T_{c}$ of ${ }^{3} \mathrm{He}(\approx 0.0026 \mathrm{~K})$ kindles the view that high-temperature superconductivity might derive from preformed realspace charged bosons rather than in the BCS state with the strongly overlapping Cooper pairs. Indeed, there is increasing experimental evidence that cuprates are bosonic superconductors [1, 2, 3]. A possible fundamental origin of such strong departure of the cuprates from conventional BCS behaviour is the unscreened (Fröhlich) EPI of the order of $1 \mathrm{eV}$ [4, 5], routinely neglected in the Hubbard $U$ and $t-J$ models of cuprate superconductors [6]. This interaction with $c$-axis polarized optical phonons is virtually unscreened because the upper limit for the out-of-plane plasmon frequency $\left(\lesssim 200 \mathrm{~cm}^{-1}[7]\right)$ in cuprates is well below the characteristic frequency of optical phonons, $\omega_{0} \approx 400-1000 \mathrm{~cm}^{-1}$. Since screening is poor, the magnetic interaction remains small compared with the Fröhlich EPI at any doping of cuprates. In order to generate a convincing theory of high-temperature superconductivity, one has to treat the long-range Coulomb repulsion and the unscreened EPI on an equal footing. When both interactions are strong compared with the kinetic energy of carriers, this Coulomb-Fröhlich model (CFM) predicts the ground state in the form of mobile small bipolarons, which bose-condense at high temperatures [4, 8, 9].

Most compelling evidence for (bi)polaronic carries in cuprate superconductors is provided by the discovery of a substantial isotope effect on the carrier mass [10] predicted for the (bi)polaronic conductors in Ref. [11]. High resolution ARPES [12, 13] provides another piece of evidence for a strong EPI in cuprates [14] apparently with c-axis-polarised optical phonons [13]. These as well as earlier optical [15], neutron scattering [16] and more recent tunnelling [17] experiments unambiguously show that the lattice vibrations play a significant though unconventional role in high temperature superconductors. Operating together with a shorter range deformation potential and molecular-type (e.g. Jahn-Teller [18]) EPIs, the Fröhlich EPI readily overcomes the Coulomb repulsion at a short distance about the lattice constant providing a non-retarded attraction to form small yet mobile bipolarons [2].

When strong EPI binds holes into intersite oxygen bipolarons [19], the chemical potential remains pinned inside the charge transfer gap, as clearly observed in the tunnelling experiments by Bozovic et al. in optimally doped $\mathrm{La}_{1.85} \mathrm{Sr}_{0.15} \mathrm{CuO}_{4}$ [20]. The bipolaron binding energy and the singlet-triplet bipolaron exchange energy are thought to be the origin of 
normal state charge and spin pseudogaps, respectively, as has been predicted by us [21] and later supported experimentally [22]. In overdoped samples carriers screen part of EPI with low frequency phonons. Hence, the bipolaron binding energy decreases [23] and the hole bandwidth increases with doping. As a result, the chemical potential enters the oxygen band in overdoped samples, so that a Fermi-level crossing could be seen in ARPPES at overdoping where mobile bipolarons coexist with the polaronic Fermi-liquid [24].

Here I briefly review a number of theoretical and experimental observations supporting the bosonic model of superconducting cuprates at variance with BCS-like and phase-fluctuation scenarios.

\section{LOW FERMI ENERGY, INDIVIDUAL PAIRING AND PARAMETER-FREE EVALUATION OF $T_{c}$}

A parameter-free estimate of the Fermi energy [25] clearly supports the real-space (i.e individual) pairing in cuprate superconductors. The band structure of cuprates is quasitwo-dimensional. Applying the parabolic approximation for the band dispersion one readily estimates the Fermi energy in 2D as $\epsilon_{F}=d \hbar^{2} c^{2} / 4 e^{2} \lambda_{a b}^{2}$, where $d$ is the distance between copper-oxygen planes, $\lambda_{a b}^{-2}=4 \pi e^{2} n / m^{*} c^{2}$ is the in-plane magnetic-field penetration depth at low temperatures, and $n, m^{*}$ are the density of hole polarons and their effective mass, respectively. Here one assumes that the 'superfluid' density at zero temperature is about the same as the normal state density of holes, as it must be in any clean superfluid [26]. The low-temperature penetration depth is unusually large, $\lambda_{a b} \gtrsim 150 \mathrm{~nm}$, in cuprates, so that the renormalised Fermi energy turns out to be surprisingly low, $\epsilon_{F} \lesssim 100 \mathrm{meV}$, rendering the Migdal-Eliashberg theory [27, 28] inadequate. In fact, $\epsilon_{F}$ is so small that the individual pairing is very likely. Such pairing will occur when the size of the pair, $r_{b}=\hbar / \sqrt{m^{*} \Delta}$ is small compared with the inter-pair separation, $r=\hbar \sqrt{\pi / m^{*} \epsilon_{F}}$, so that the condition for real-space pairing is $\epsilon_{F} \lesssim \pi \Delta$. Experimentally measured pseudogaps of many cuprates are about $50 \mathrm{meV}$ or larger. If one accepts that the pseudogap is about half of the pair binding energy, $\Delta / 2,[21]$ then the condition for real-space pairing is well satisfied in most cuprates (typically $r_{b} \approx 0.2-0.4 \mathrm{~nm}$ ).

When bipolarons are small so that pairs do not overlap, the pairs can form a Bose-Einstein

condensate (BEC) [2]. Recent Quantum Monte Carlo simulations of CFM show that with 
realistic values of EPI coupling constant, $\lambda \simeq 1$, and high optical phonon frequencies one can avoid overlap of pairs and get a very high bose-condensation temperature $T_{B E C}[9]$. Actually $T_{B E C}$ fits the experimental $T_{c}$ in a great number of cuprates without any fitting parameters [29]. In contrast with Ref. [30] our expression for $T_{B E C}$ involves not only the in-plane, $\lambda_{a b}$, but also the out-of-plane, $\lambda_{c}$, magnetic field penetration depth, and the normal state Hall ratio $R_{H}$ just above the transition,

$$
T_{B E C} \approx 1.64\left(\frac{e R_{H}}{\lambda_{a b}^{4} \lambda_{c}^{2}}\right)^{1 / 3}
$$

Here $T_{c}$ is measured in Kelvin, $e R_{H}$ in $\mathrm{cm}^{3}$ and $\lambda$ in $\mathrm{cm}$. Since all quantities in Eq.(1) are measurable, the bipolaron theory provides the parameter-free expression, which unambiguously tells us how near cuprates are to the BEC regime. Its comparison with the experimental $T_{c}$ of more than 30 different cuprates, where both $\lambda_{a b}$ and $\lambda_{c}$ have been measured along with $R_{H}\left(T_{c}+0\right)$, show that $T_{B E C}$ fits experimental values within an experimental error bar for the penetration depths.

One can argue that due to a large anisotropy cuprates may belong to the $2 \mathrm{D}$ ' $X Y$ ' universality class with the Kosterlitz-Thouless (KT) superfluid critical temperature $T_{K T}$ of the Cooper pairs [31]. The KT transition temperature is expressed through the in-plane penetration depth alone as $T_{K T} \approx d \hbar^{2} c^{2} / 4 k_{B} \pi e^{2} \lambda_{a b}^{2}$. It turns out significantly higher than the experimental values in many cuprates. There are also quite a few samples with about the same $\lambda_{a b}$ and about the same inter-plane distance $d$, but with very different values of $T_{c}$, which makes the KT scenario unviable. On the contrary, our parameter-free fit of the experimental critical temperature and the critical behavior (see below) favor $3 D$ BEC of charged bosons as the mechanism of high $\mathrm{T}_{c}$ rather than any low-dimensional phasefluctuation scenario.

\section{UPPER CRITICAL FIELD AND THE NORMAL STATE NERNST EFFECT}

The state of bipolarons above the resistive $T_{c}$ is perfectly "normal" in the sense that the off-diagonal order parameter (i.e. the Bogoliubov-Gor'kov anomalous average $\mathcal{F}\left(\mathbf{r}, \mathbf{r}^{\prime}\right)=$ $\left\langle\psi_{\downarrow}(\mathbf{r}) \psi_{\uparrow}\left(\mathbf{r}^{\prime}\right\rangle\right)$ is zero (here $\psi_{\downarrow, \uparrow}(\mathbf{r})$ annihilates electrons with spin $\downarrow, \uparrow$ at the point $\mathbf{r}$ ).

On the contrary $\mathcal{F}\left(\mathbf{r}, \mathbf{r}^{\prime}\right)$ remains nonzero in the phase fluctuation scenario [31] with vortexes in the normal state above $T_{c}[32,33]$. We have noticed that the phase fluctuation 
scenario is not compatible with extremely sharp resistive transitions at $T_{c}$ in high-quality underdoped, optimally and overdoped cuprates, and with the insulating-like in-plane resistivity of underdoped cuprates in high magnetic fields [34, 35]. For example, the in-plane and out-of-plane resistivity of $B i-2212$, where the anomalous Nernst signal has been measured [32], is perfectly "normal" above $T_{c}$, showing only a few percent positive or negative magnetoresistance [36], explained with bipolarons [37].

Both in-plane and out-of-plane resistive transitions of high-quality samples remain sharp in the magnetic field providing a reliable determination of the genuine upper critical field, $H_{c 2}(T)$. Many high magnetic field studies revealed a non-BCS upward curvature of $H_{c 2}(T)$ (for review see [38]) with a non-linear temperature dependence in the vicinity of $T_{c}$ in many cuprates and some other unconventional superconductors, Fig.1. If unconventional superconductors are in the 'bosonic' limit, such unusual critical fields are expected in accordance with the theoretical prediction for BEC of charged bosons in the external magnetic field [39].

Also the bipolaron theory accounts for the anomalous Nernst signal, and the insulatinglike in-plane resistivity of underdoped cuprates [34, 35] as observed [32, 33, 40, 41]. The transverse Nernst-Ettingshausen effect (here the Nernst effect) is the appearance of a transverse electric field $E_{y}$ perpendicular to the magnetic filed and to a temperature gradient, $\nabla_{x} T$. When bipolarons are formed the chemical potential is negative in the normal state. It is found in the impurity band just below the mobility edge at $T>T_{c}$. Carriers, localised below the mobility edge contribute to the longitudinal transport together with the itinerant carriers in extended states above the mobility edge. Importantly the contribution of localised carriers adds to the contribution of itinerant carriers to produce a large Nernst signal, $e_{y} \equiv-E_{y} / \nabla_{x} T$, while it reduces the thermopower $S$ and the Hall angle $\Theta$. This unusual "symmetry breaking" is at variance with conventional metals where the familiar "Sondheimer" cancelation [42] makes $e_{y}$ much smaller than $S \tan \Theta$ because of the electronhole symmetry near the Fermi level. Such behaviour originates in the "sign" (or " $p-n$ ") anomaly of the Hall conductivity of localised carriers. The sign of their Hall effect is often opposite to that of the thermopower as observed in many amorphous semiconductors [43].

Hence the bipolaron model can account for a low value of $S \tan \Theta$ compared with a large value of $e_{y}$ in some underdoped cuprates [32, 41] due to the sign anomaly. Near the mobility edge $S \propto T$ as in conventional amorphous semiconductors [44], so that $S \tan \Theta \propto T / \rho(T)$ 


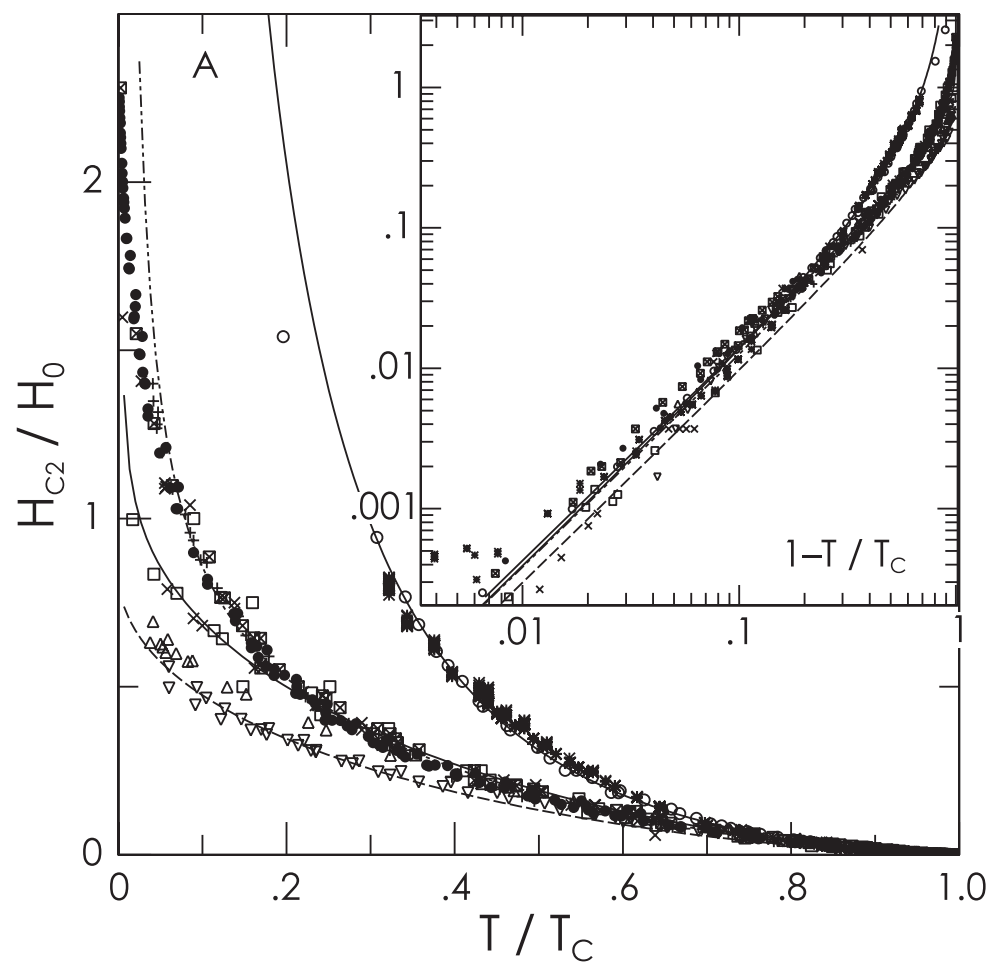

FIG. 1: Resistive upper critical field [38] (determined at 50\% of the transition) of cuprates, spinladders and organic superconductors scaled according to the Bose-Einstein condensation field of charged bosons [39], $H_{c 2}(T) \propto\left[b(1-t) / t+1-t^{1 / 2}\right]^{3 / 2}$ with $t=T / T_{c}$. The parameter $b$ is proportional to the number of delocalised bosons at zero temperature, $b$ is 1 (solid line), 0.02 (dashed-dotted line), 0.0012 (dotted line), and 0 (dashed line). The inset shows a universal scaling of the same data near $T_{c}$ on the logarithmic scale. Symbols correspond to $T l-2201(\bullet)$, $\mathrm{La}_{1.85} \mathrm{Sr}_{0.15} \mathrm{CuO}_{4}(\triangle), \mathrm{Bi}-2201(\times), \mathrm{Bi}-2212(*), \mathrm{YBa}_{2} \mathrm{Cu} \mathrm{H}_{3} \mathrm{O}_{6+x}(\mathrm{\circ}), \mathrm{La}_{2-x} \mathrm{Ce} \mathrm{e}_{x} \mathrm{CuO} \mathrm{O}_{4-y}(\square)$, $\mathrm{Sr}_{2} \mathrm{Ca}_{12} \mathrm{Cu}_{24} \mathrm{O}_{41}(+)$, and Bechgaard salt organic superconductor $(\nabla)$.

and $e_{y} \propto\left(1-T / T_{1}\right) / \rho(T)$.

According to our earlier suggestion [45] the insulating-like low-temperature dependence of $\rho(T)$ in underdoped cuprates originates from the elastic scattering of nondegenerate itinerant carriers by charged impurities. The relaxation time of nondegenerate carriers depends on temperature as $\tau \propto T^{-1 / 2}$ for scattering by short-range deep potential wells, and as $T^{1 / 2}$ for shallow wells [45]. Combining both scattering rates one obtains $\rho=\rho_{0}\left[\left(T / T_{2}\right)^{1 / 2}+\right.$ $\left.\left(T_{2} / T\right)^{1 / 2}\right]$, which fits extremely well the experimental insulating-like normal state resistivity of underdoped $\mathrm{La}_{1.94} \mathrm{Sr}_{0.06} \mathrm{CuO}_{4}$, Fig. 2, with $\rho_{0}=0.236 \mathrm{~m} \Omega \cdot \mathrm{cm}$ and $T_{2}=44.6 \mathrm{~K}$. Then 


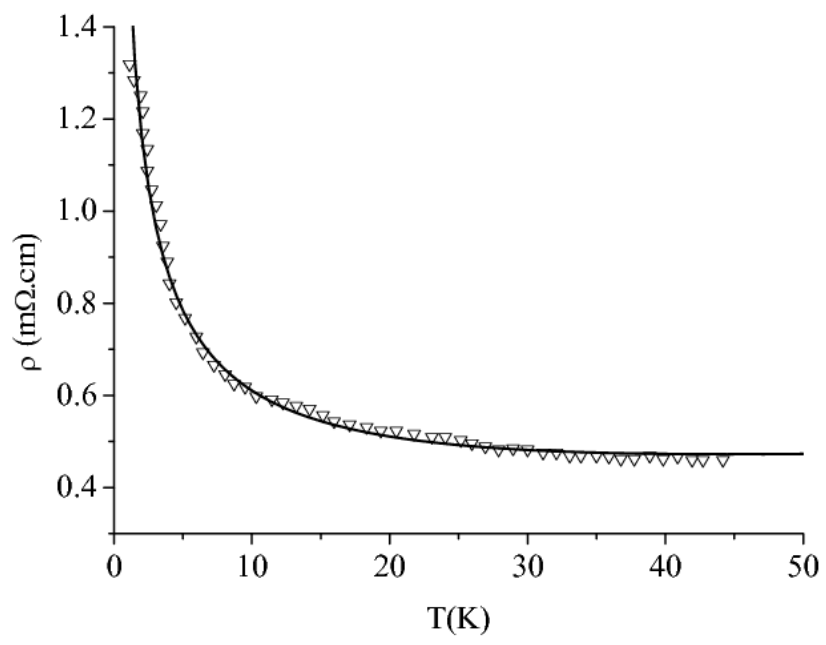

FIG. 2: Normal state in-plane resistivity of underdoped $\mathrm{La}_{1.94} \mathrm{Sr}_{0.06} \mathrm{CuO}_{4}$ (triangles [40]) as revealed in the field $B=12$ Tesla and compared with the bipolaron theory [24] (solid line).

$S \tan \Theta$ and $e_{y}$ can be parameterized as

$$
S \tan \Theta=e_{0} \frac{\left(T / T_{2}\right)^{3 / 2}}{1+T / T_{2}}
$$

and

$$
e_{y}(T, B)=e_{0} \frac{\left(T_{1}-T\right)\left(T / T_{2}\right)^{1 / 2}}{T_{2}+T}
$$

where $T_{1}$ and $e_{0}$ are temperature independent.

In spite of all simplifications, the model describes remarkably well both $S \tan \Theta$ and $e_{y}$, Fig 3. measured in $\mathrm{La}_{1.94} \mathrm{Sr}_{0.06} \mathrm{CuO}_{4}$ with a single fitting parameter, $T_{1}=50 \mathrm{~K}$ using the experimental $\rho(T)$. The constant $e_{0}=2.95 \mu \mathrm{V} / \mathrm{K}$ scales the magnitudes of $S \tan \Theta$ and $e_{y}$. The magnetic field $B=12$ Tesla destroys the superconducting state of the low-doped $\mathrm{La}_{1.94}$ $\mathrm{Sr}_{0.06} \mathrm{CuO}_{4}$ down to $2 \mathrm{~K}$, Fig.2, so any residual superconducting order above $2 \mathrm{~K}$ (including vortexes) is clearly ruled out, while the Nernst signal, is remarkably large. The coexistence of the large Nernst signal and a nonmetallic resistivity is in sharp disagreement with the vortex scenario, but in agreement with our model.

\section{HALL-LORENZ NUMBER AND NORMAL STATE DIAMAGNETISM}

The measurements of the Righi-Leduc effect provides further evidence for charged bosons above $T_{c}$ [46]. The effect describes transverse heat flow resulting from a perpendicular 

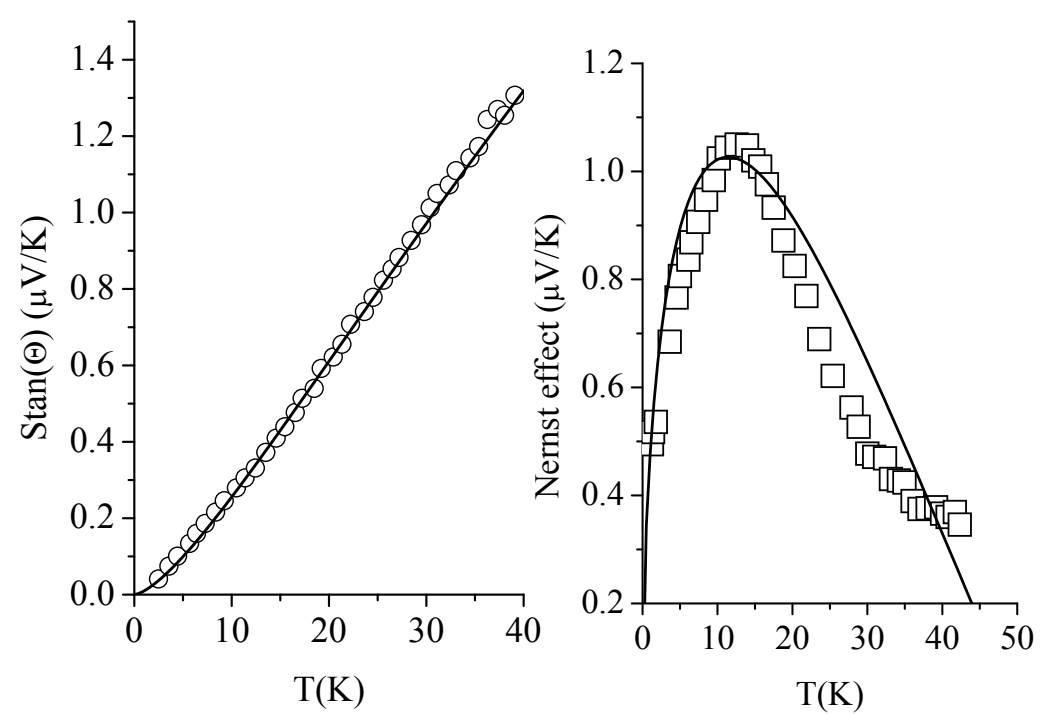

FIG. 3: $S \tan \Theta$ (circles [41] ) and the Nernst effect $e_{y}$ (squares [40]) of underdoped La1.94 $\mathrm{Sr}_{0.06} \mathrm{CuO}_{4}$ at $B=12$ Tesla compared with the bipolaron theory (solid lines) [35].

temperature gradient in an external magnetic field, which is a thermal analog of the Hall effect. Using the effect the "Hall-Lorenz" electronic number, $L_{H}=\left(e / k_{B}\right)^{2} \kappa_{x y} /\left(T \sigma_{x y}\right)$ has been directly measured [47] in $\mathrm{YBa}_{2} \mathrm{Cu}_{3} \mathrm{O}_{6.95}$ and $\mathrm{YBa}_{2} \mathrm{Cu}_{3} \mathrm{O}_{6.6}$ since transverse thermal $\kappa_{x y}$ and electrical $\sigma_{x y}$ conductivities involve no phonons. The experimental $L_{H}(T)$ showed a quasi-linear temperature dependence above the resistive $T_{c}$, which strongly violates the Wiedemann-Franz (WF) law. Remarkably, the measured value of $L_{H}$ just above $T_{c}$ turned out about the same as predicted by the bipolaron theory [48], $L=0.15 L_{0}$, where $L_{0}=\pi^{2} / 3$ is the conventional Sommerfeld value. The breakdown of the WF law revealed in the RighiLeduc effect [47] has been explained by a temperature-dependent contribution of thermally excited single polarons to the transverse magneto-transport [46].

Most surprisingly the torque magnetometery [49, 50] uncovered a diamagnetic signal somewhat above $T_{c}$ which increases in magnitude with applied magnetic field. It has been linked with the Nernst signal and mobile vortexes in the normal state of cuprates [33]. However, apart from the inconsistences mentioned above, the vortex scenario of the normalstate diamagnetism is internally inconsistent. Accepting the vortex scenario and fitting the magnetization data in $B i-2212$ with the conventional logarithmic field dependence [33], one obtains surprisingly high upper critical fields $H_{c 2}>120$ Tesla and a very large 
Ginzburg-Landau parameter, $\kappa=\lambda / \xi>450$ even at temperatures close to $T_{c}$. The inplane low-temperature magnetic field penetration depth is $\lambda=200 \mathrm{~nm}$ in optimally doped $B i-2212$ (see, for example [51]). Hence the zero temperature coherence length $\xi$ turns out about the lattice constant, $\xi=0.45 \mathrm{~nm}$, or even smaller. Such a small coherence length rules out the "preformed Cooper pairs" [31], since the pairs are virtually not overlapped at any size of the Fermi surface in $B i-2212$. Moreover the magnetic field dependence of $M(T, B)$ at and above $T_{c}$ is entirely inconsistent with what one expects from a vortex liquid. While $-M(B)$ decreases logarithmically at temperatures well below $T_{c}$, the experimental curves [33, 49, 50] clearly show that $-M(B)$ increases with the field at and above $T_{c}$, just opposite to what one could expect in the vortex liquid. This significant departure from the London liquid behavior clearly indicates that the vortex liquid does not appear above the resistive phase transition (see also Ref. [49]).

On the contrary the bipolaron theory, which predicted the unusual upper critical field [39], Fig. 1, quantitatively accounts for the normal state diamagnetism of cuprates as well [52]. When the magnetic field is applied perpendicular to the copper-oxygen plains the quasi-2D bipolaron energy spectrum is quantized as $E_{\alpha}=\hbar \omega(p+1 / 2)+2 t_{c}\left[1-\cos \left(K_{z} d\right)\right]$, where $\alpha$ comprises $p=0,1,2, \ldots$ and in-plane $K_{x}$ and out-of-plane $K_{z}$ center-of-mass quasi-momenta, $t_{c}$ and $d$ are the hopping integral and the lattice period perpendicular to the planes, and $\omega=2 e B / c \sqrt{m_{x}^{* *} m_{y}^{* *}}$. The bipolaron spectrum consists of two degenerate branches, socalled " $x "$ and " $y$ " bipolarons [4] with anisotropic in-plane bipolaron masses $m_{x}^{* *}$ and $m_{y}^{* *}$. Expanding the Bose-Einstein distribution function in powers of $\exp \left[(\mu-E) / k_{B} T\right]$ with the negative chemical potential $\mu$ one can after summation over $p$ readily obtain the boson density,

$$
n_{b}=\frac{2 e B}{\pi \hbar c d} \sum_{r=1}^{\infty} I_{0}\left(2 t_{c} r / k_{B} T\right) \frac{\exp \left[\left(\mu-\hbar \omega / 2-2 t_{c}\right) r / k_{B} T\right]}{1-\exp \left(-\hbar \omega r / k_{B} T\right)},
$$

and the magnetization,

$$
\begin{aligned}
& M(T, B)=-n_{b} \mu_{b}+\frac{2 e k_{B} T}{\pi \hbar c d} \sum_{r=1}^{\infty} I_{0}\left(2 t_{c} r / k_{B} T\right) \times \\
& \frac{\exp \left[\left(\mu-\hbar \omega / 2-2 t_{c}\right) r / k_{B} T\right]}{1-\exp \left(-\hbar \omega r / k_{B} T\right)}\left(\frac{1}{r}-\frac{\hbar \omega \exp \left(-\hbar \omega r / k_{B} T\right)}{k_{B} T\left[1-\exp \left(-\hbar \omega r / k_{B} T\right)\right]}\right) .
\end{aligned}
$$

Here $\mu_{b}=e \hbar / c \sqrt{m_{x}^{* *} m_{y}^{* *}}$, and $I_{0}(x)$ is the modified Bessel function. At low temperatures $T \rightarrow 0$ Schafroth's result [53] is recovered, $M(0, B)=-n_{b} \mu_{b}$. The magnetization of charged bosons is field-independent at low temperatures. At high temperatures, $T \gg T_{c}$ the chem- 


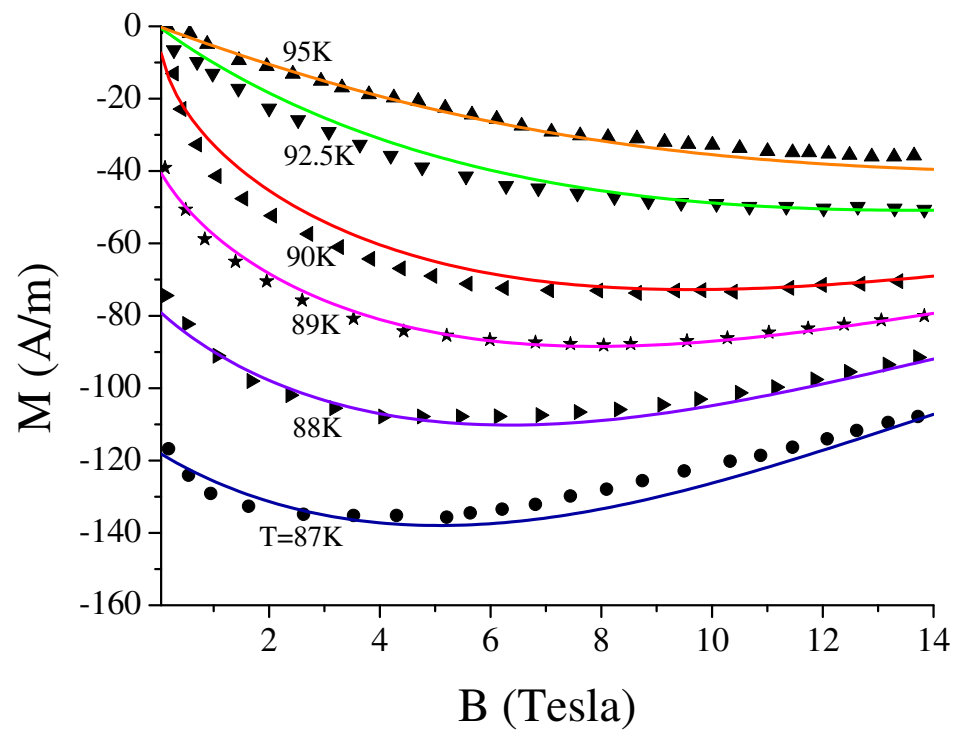

FIG. 4: Diamagnetism of optimally doped Bi-2212 (symbols) [33] compared with the magnetization of charged bosons [52] near and above $T_{c}$ (lines).

ical potential has a large magnitude, and we can keep only the terms with $r=1$ to obtain $M(T, B)=-n_{b} \mu_{b} \hbar \omega /\left(6 k_{B} T\right)$ at $T \gg T_{c} \gg \hbar \omega / k_{B}$, which is the familiar Landau orbital diamagnetism of nondegenerate carriers. Here $T_{c}$ is the Bose-Einstein condensation temperature $T_{c}=3.31 \hbar^{2}\left(n_{b} / 2\right)^{2 / 3} /\left(m_{x}^{* *} m_{y}^{* *} m_{c}^{* *}\right)^{1 / 3}$, with $m_{c}=\hbar^{2} / 2\left|t_{c}\right| d^{2}$.

Comparing with the experimental data one has to take into account a temperature and field depletion of singlets due to their thermal excitations into spin-split triplets and singlepolaron states by allowing for some temperature and field dependences of the singlet bipolaron density, $n_{b}(T, B)=n_{c}\left[1-\alpha \tau-\left(B / B^{*}\right)^{2}\right]$ (here $\left.\tau=T / T_{c}-1\right)$. As a result, $M(T, B)$ of charged bosons fits remarkably well the experimental orbital magnetization of optimally doped Bi-2212, Fig. 4, with $n_{c} \mu_{b}=2100 \mathrm{~A} / \mathrm{m}, T_{c}=90 \mathrm{~K}, \alpha=0.62$ and $B^{*}=56$ Tesla.

On the other hand the experimental data, Fig. 4, are in disagreement with the phasefluctuation scenario [31, 33]. Indeed, a critical exponent $\delta=\ln B / \ln |M(T, B)|$ for $B \rightarrow 0$, in the charged Bose gas is dramatically different from the KT critical exponent. While the $\mathrm{KT} \delta$ is about 1 above $T_{K T}$, charged bosons have strongly temperature dependent $\delta(T)>1$, which is very close to the experimental $\delta(T)$ [52]. 


\section{GIANT PROXIMITY EFFECT}

Several groups reported that in the Josephson cuprate $S N S$ junctions supercurrent can run through normal $N$-barriers as thick as $10 \mathrm{~nm}$ or even thicker in a strong conflict with the standard theoretical picture. Using the advanced molecular beam epitaxy, Bozovic et al. [54] proved that this giant proximity effect (GPE) is intrinsic, rather than caused by any inhomogeneity of the barrier such as stripes, superconducting "islands", etc.. Hence GPE defies the conventional explanation, which predicts that the critical current should exponentially decay with the characteristic length of about the coherence length, which is $\xi \lesssim 1 \mathrm{~nm}$ in the cuprates.

This unusual effect can be broadly understood as the Bose-Einstein condensate tunnelling into a cuprate semiconductor [55]. The condensate wave function, $\psi(z)$, is described by the Gross-Pitaevskii (GP) equation. In the superconducting region, $z<0$, near the $S N$ boundary at $z=0$, the GP equation is

$$
\frac{\hbar^{2}}{2 m^{* *}} \frac{d^{2} \psi(z)}{d z^{2}}=\left[V|\psi(z)|^{2}-\mu\right] \psi(z),
$$

where $V$ is a short-range repulsion of bosons, and $m^{* *}$ is the boson mass along the direction of tunnelling $z$. Deep inside the superconductor $|\psi(z)|^{2}=n_{s}$ so that $\mu=V n_{s}$, where the condensate density $n_{s}$ is about half of the hole density, if the temperature is well below $T_{c}$. Then the coherence length is $\xi=\hbar /\left(2 m^{* *} n_{s} V\right)^{1 / 2}$ from Eq.([6).

If the normal barrier, at $z>0$, is an underdoped cuprate semiconductor above its transition temperature, the chemical potential $\mu$ lies below the quasi-2D bosonic band by some energy $\epsilon$ given by

$$
\epsilon(T) \leqslant-k_{B} T \ln \left(1-e^{-T_{0} / T}\right)
$$

which is exponentially small at $T_{c}^{\prime}<T \ll T_{0}$ turning into zero at $T=T_{c}^{\prime}$. Here $T_{c}^{\prime} \approx$ $T_{0} / \ln \left(k_{B} T_{0} / 2 t_{c}\right)$, is the transition temperature of the barrier, and $T_{0}=\pi \hbar^{2} n^{\prime} d / k_{B} m \gg$ $T_{c}^{\prime} \gg t_{c} / k_{B}$. The GP equation in the barrier is written as

$$
\frac{\hbar^{2}}{2 m^{* *}} \frac{d^{2} \psi(z)}{d z^{2}}=\left[V|\psi(z)|^{2}+\epsilon\right] \psi(z)
$$

It predicts the occurrence of a new length scale, $\hbar / \sqrt{2 m^{* *} \epsilon(T)}$. In a wide temperature range $T_{c}^{\prime}<T<T_{0}$, this length turns out much larger than the zero-temperature coherence length, $\xi$, since $\epsilon(T)$ in Eq.(7) could be very small, which explains GPA. The physical reason 
why the quasi-2D bosons display a large normal-state coherence length, whereas 3D Bosesystems (or any-D Fermi-systems) at the same values of parameters do not, originates in the large density of states (DOS) near the band edge of two-dimensional bosons compared with 3D DOS. Since DOS is large, the chemical potential is pinned near the edge with the exponentially small magnitude, $\epsilon(T)$, at $T<T_{0}$. Importantly if the barrier is undoped ( $\left.n^{\prime} \rightarrow 0\right) \epsilon(T)$ becomes large, $\epsilon(T) \propto \ln \left(1 / n^{\prime}\right) \rightarrow \infty$, for any finite temperature $T$. In this case the current should exponentially decay with the characteristic length smaller that $\xi$, as is also experimentally observed [20].

\section{SUMMARY}

A first proposal for high temperature superconductivity, made by Ogg Jr in 1946 [56], already involved real-space pairing of individual electrons into bosonic molecules with zero total spin. This idea was further developed as a natural explanation of conventional superconductivity by Schafroth [53] and Butler and Blatt [57]. The Ogg-Schafroth picture was practically forgotten because it neither accounted quantitatively for the critical behavior of conventional superconductors, nor did it explain the microscopic nature of attractive forces which could overcome the Coulomb repulsion between two electrons constituting a pair. On the contrary highly successful for low- $T_{c}$ metals and alloys the BCS theory, where two electrons were indeed correlated, but at a very large distance of about $10^{3}$ times of the average inter-electron spacing, led many researchers to believe that cuprate superconductors should also be "BCS-like" (maybe with strong phase fluctuations).

However, by extending the BCS theory towards the strong EPI, a charged Bose liquid of small bipolarons was predicted by us [58] with a further prediction that high $T_{c}$ should exist in the crossover region of the EPI strength from the BCS-like to bipolaronic superconductivity [59]. Later on we have shown that the unscreened Fröhlich EPI combined with the strong Coulomb repulsion provides superlight small bipolarons, which are several orders lighter than the Holstein bipolarons [4, 8, 9]. The bipolaron theory predicted such key features of cuprate superconductors as anomalous upper critical fields [39], spin and charge pseudogaps [21], and anomalous isotope effects [11] later observed experimentally (for review see [1, 2]). The strong EPI has been experimentally established in cuprates beyond any reasonable doubt. 
We believe that the following conditions are responsible for the high-temperature superconductivity in cuprates [9]: (a) The parent compounds are ionic insulators with light oxygen ions to form high-frequency optical phonons, (b) The structure is quasi two-dimensional to ensure poor screening of EPI with c-axis polarized phonons, (c) There is strong on-site Coulomb repulsion to form intersite mobile bipolarons rather than strongly localised on-site pairs, (d) There are moderate carrier densities to keep the system of small bipolarons close to the dilute regime.

Here I have shown that the normal-state diamagnetism, the Nernst, thermal Hall and giant proximity effects provide further evidence for a genuine Bose-Einstein condensation of real-space lattice bipolarons in cuprates.

I would like to thank Peter Edwards, Jim Hague, Viktor Kabanov, Pavel Kornilovitch, John Samson and Vladimir Zavaritsky for collaboration and helpful discussions, and to acknowledge EPSRC (UK) (grant numbers EP/C518365/1 and EP/D07777X/1).

[1] A. S. Alexandrov and N. F. Mott, Rep. Prog. Phys. 57, 1197 (1994).

[2] A. S. Alexandrov, Theory of Superconductivity: From Weak to Strong Coupling (IoP Publishing, Bristol, 2003).

[3] P. P. Edwards, C. N. R. Rao, N. Kumar, and A. S. Alexandrov, ChemPhysChem 7, 2015 (2006).

[4] A. S. Alexandrov, Phys. Rev. B 53, 2863 (1996).

[5] A. S. Alexandrov and A. M. Bratkovsky, Phys. Rev. Lett. 84, 2043 (2000).

[6] P. W. Anderson, P. A. Lee, M. Randeria, T. M. Rice, N. Trivedi, and F. C. Zhang, J. Phys.: Condens. Matter 16, R755 (2004).

[7] J. H. Kim, B. J. Feenstra, H. S. Somal, D. van der Marel, W. Y. Lee, A. M. Gerrits, and A. Wittlin, Phys. Rev. B49, 13065 (1994).

[8] A. S. Alexandrov and P. E. Kornilovitch, J. Phys.: Condens. Matter 14, 5337 (2002).

[9] J. P. Hague, P. E. Kornilovitch, J. H. Samson, and A. S. Alexandrov, cond-mat/0606036.

[10] G. Zhao and D. E. Morris, Phys. Rev. B 51, 16487 (1995); G.-M. Zhao, M. B. Hunt, H. Keller, and K. A. Müller, Nature 385, 236 (1997); R. Khasanov, D. G. Eshchenko, H. Luetkens, E. Morenzoni, T. Prokscha, A. Suter, N. Garifianov, M. Mali, J. Roos, K. Conder, and H. Keller 
Phys. Rev. Lett. 92, 057602 (2004).

[11] A. S. Alexandrov, Phys. Rev. B 46, 14932 (1992).

[12] A. Lanzara, P. V. Bogdanov, X. J. Zhou, S. A. Kellar, D. L. Feng, E. D. Lu, T. Yoshida, H. Eisaki, A. Fujimori, K. Kishio, J. I. Shimoyana, T. Noda, S. Uchida, Z. Hussain, Z. X. Shen, Nature 412, 510 (2001); G. H. Gweon, T. Sasagawa, S. Y. Zhou, J. Craf, H. Takagi, D. H. Lee, A. Lanzara, Nature 430, 187 (2004).

[13] W. Meevasana, N. J. C. Ingle, D. H. Lu, J. R. Shi, F. Baumberger, K. M. Shen, W. S. Lee, T. Cuk, H. Eisaki, T. P. Devereaux, N. Nagaosa, J. Zaanen, and Z.-X. Shen, Phys. Rev. Lett. 96, 157003 (2006).

[14] P. B. Allen, Nature 412, 494 (2001).

[15] D. Mihailovic, C. M. Foster, K. Voss, and A. J. Heeger, Phys. Rev. B42, 7989 (1990); P. Calvani, M. Capizzi, S. Lupi, P. Maselli, A. Paolone, P. Roy, S. W. Cheong, W. Sadowski, and E. Walker, Solid State Commun. 91, 113 (1994); R. Zamboni, G. Ruani, A. J. Pal, and C. Taliani, Solid St. Commun. 70, 813 (1989).

[16] T. R. Sendyka, W. Dmowski, and T. Egami, Phys. Rev. B51, 6747 (1995); T. Egami, J. Low Temp. Phys. 105, 791 (1996).

[17] J. Lee, K. Fujita, K. McElroy, J. A. Slezak, M. Wang, Y. Aiura, H. Bando, M. Ishikado, T. Masui, J. X. Zhu, A. V. Balatsky, H. Eisaki, S. Uchida, and J. C. Davis, Nature 442, 546 (2006).

[18] K. A. Müller, Physica C 341, 11 (2000).

[19] There is no lattice instability in the strong EPI limit, $\lambda>1$, since the phonon frequency renormalisation, $\delta \omega_{0}$, is small as $\delta \omega_{0} / \omega_{0} \lesssim 1 / \lambda^{2}$ at any polaron density [A. S. Alexandrov, Phys. Rev. B 46, 2838 (1992)].

[20] I. Bozovic, G. Logvenov, M. A. J. Verhoeven, P. Caputo, E. Goldobin, and T. H. Geballe, Nature (London) 422, 873 (2003).

[21] A. S. Alexandrov, J. Low Temp. Phys. 87, 721 (1992); A. S. Alexandrov and N. F. Mott, J. Supercond (US), 7, 599 (1994).

[22] D. Mihailovic, V. V. Kabanov, K. Zagar, and J. Demsar, Phys. Rev. B60, 6995 (1999) and references therein.

[23] A. S. Alexandrov, V. V. Kabanov, and N. F. Mott, Phys. Rev. Lett. 77, 4796 (1996).

[24] A. S. Alexandrov, in Studies in High Temperature Superconductors, ed. A. V. Narlikar (Nova 
Science Pub., NY 2006), Golden Jubilee volume 50, pp. 1-69.

[25] A. S. Alexandrov, Physica C 363, 231 (2001).

[26] A. J. Leggett, Physica Fennica 8, 125 (1973); J Stat. Phys. 93, 927 (1998); V. N. Popov Functional Integrals and Collective Excitations (Cambridge University Press, Cambridge (1987)).

[27] A. B. Migdal, Zh. Eksp. Teor. Fiz. 34, 1438 (1958)[Sov. Phys. JETP 7, 996 (1958)].

[28] G. M. Eliashberg, Zh. Eksp. Teor. Fiz. 38, 966 (1960); 39, 1437 (1960) [Sov. Phys. JETP 11, $696 ; 12,1000(1960)]$.

[29] A. S. Alexandrov, Phys. Rev. Lett. 82, 2620 (1999); A. S. Alexandrov and V. V. Kabanov, Phys. Rev. B 5913628 (1999).

[30] Y. J. Uemura, Polarons and Bipolarons in High-Tc Superconductors and Related Materials (eds. E. K. H. Salje, A. S. Alexandrov, and W. Y. Liang, Cambridge University Press, Cambridge) p. 453 (1995).

[31] V. J. Emery and S. A. Kivelson, Nature, 374, 434 (1995).

[32] Z.A. Xu, N. P. Ong, Y. Wang, T. Kakeshita, and S. Uchida, Nature (London) 406, 486 (2000); N. P. Ong and Y. Wang, Physica C408, 11 (2004) and references therein.

[33] Y. Wang, L. Li, M. J. Naughton, G.D. Gu, S. Uchida, and N. P. Ong, Phys. Rev. Lett. 95, 247002 (2005); L. Li, Y. Wang, M. J. Naughton, S. Ono, Y. Ando, and N. P. Ong, Europhys. Lett. 72, 451(2005); Y. Wang, L. Li, and N. P. Ong, Phys. Rev. B 73, 024510 (2006).

[34] A. S. Alexandrov and V. N. Zavaritsky, Phys. Rev. Lett. 93, 217002 (2004).

[35] A. S. Alexandrov, Phys. Rev. Lett. 95, 259704 (2005).

[36] V. N. Zavaritsky and A. S. Alexandrov, Phys. Rev. B71, 012502 (2005).

[37] V. N. Zavaritsky, J. Vanacken, V. V. Moshchalkov, and A. S. Alexandrov, Eur. Phys. J. B 42, 367 (2004).

[38] V. N. Zavaritsky, V. V. Kabanov, A. S. Alexandrov, Europhys. Lett. 60, 127 (2002).

[39] A. S. Alexandrov, Doctoral Thesis MEPHI (Moscow, 1984); Phys. Rev. B 48, 10571 (1993).

[40] C. Capan, K. Behnia, J. Hinderer, A. G. M. Jansen, W. Lang, C. Marcenat, C. Martin, and J. Flouquet, Phys. Rev. Lett. 88, 056601 (2002).

[41] C. Capan and K. Behnia, Phys. Rev. Lett. 95, 259703 (2005).

[42] E. H. Sondheimer, Proc. Roy. Soc. 193, 484 (1948).

[43] S. R. Elliot, Physics of amorphous materials (Longman, New York, 1983), pp. 222-225.

[44] M. Cutler and N. F. Mott, Phys. Rev. 181, 1336 (1969). 
[45] A. S. Alexandrov, Phys. Lett. A 236, 132 (1997).

[46] K. K. Lee, A. S. Alexandrov, and W. Y. Liang, Phys. Rev. Lett. 90, 217001 (2003); Eur. Phys. J. B30, 459 (2004); A. S. Alexandrov, Phys. Rev. B 73, 100501 (2006).

[47] Y. Zhang, N. P. Ong, Z. A. Xu, K. Krishana, R. Gagnon and L. Taillefer, Phys. Rev. Lett. 84, 2219 (2000), and unpublished.

[48] A. S. Alexandrov and N. F. Mott, Phys. Rev. Lett. 71, 1075 (1993).

[49] C. Bergemann, A. W. Tyler, A. P. Mackenzie, J. R. Cooper, S. R. Julian, and D. E. Farrel, Phys. Rev. B 57, 14387 (1998).

[50] M. J. Naughton, Phys. Rev. B 61, 1605 (2000).

[51] J. L. Tallon, J. W. Loram, J. R. Cooper, C. Panagopoulos, and C. Bernhard, Phys. Rev. B 68, 180501(R) (2003).

[52] A. S. Alexandrov, Phys. Rev. Lett. 96, 147003 (2006).

[53] M. R. Schafroth, Phys. Rev. 100, 463 (1955).

[54] I. Bozovic, G. Logvenov, M. A. J. Verhoeven, P. Caputo, E. Goldobin, and M. R. Beasley, Phys. Rev. Lett. 93, 157002 (2004), and references therein.

[55] A. S. Alexandrov, cond-mat/0607770.

[56] R. A. Ogg Jr., Phys. Rev. 69, 243 (1946).

[57] J. M. Blatt and S. T. Butler, Phys. Rev. 100, 476 (1955).

[58] A. S. Alexandrov and J. Ranninger, Phys. Rev. B 231796 (1981), ibid 24, 1164 (1981).

[59] A. S. Alexandrov, Russ. J. Phys. Chem. 57167 (1983). 